\title{
Complete Pericardial Agenesis in a Pregnant Patient: A Clinical Dilemma
}

\author{
Carolina de Souza Galvão, ${ }^{1}$ Sterffeson Lamare Lucena de Abreu, ${ }^{1}$ Magda Luciene de Sousa Carvalho, ${ }^{\circledR}$ \\ Gardenia Nogueira e Silva, ${ }^{(\oplus)}$ Júlio Cezar Uchoa Serra, ${ }^{1 \oplus}$ Ana Bárbara Silva dos Santos Leite ${ }^{1}$ \\ Hospital Universitário da Universidade Federal do Maranhão, São Luís, MA - Brazil
}

\begin{abstract}
Pericardial agenesis is a rare congenital anomaly found predominantly in men, and its complete form is extremely rare and difficult to diagnose. This report describes the case of a pregnant patient with complete pericardial agenesis in which mode of delivery and sterilization raised debate among specialists.

\section{Introduction}

Pericardial agenesis, or absence of pericardium, is a rare congenital anomaly found predominantly in men compared to women (3:1 - three-to-one ratio) $)^{1,2}$ and has an estimated prevalence from 0.002 to $0.044 \%$ when associated with other heart or lung diseases. Its isolated presentation has an undefined prevalence, due to probable underdiagnosis related to asymptomatic presentations or to atypical symptoms. ${ }^{3,4}$

This is the report of an extremely rare case of a 30-yearold pregnant woman with complete pericardial agenesis in which mode of delivery and sterilization raised debate in our service, considering the lack of evidence on management practices for this condition.

\section{Case report}

A 37-week pregnant woman aged 30 years with complete pericardial agenesis, diagnosed 10 years ago, was referred from the cardiology department to the maternity ward. She had no other known congenital or acquired diseases. She complained of palpitations and dyspnea on moderate exertion.

\section{Keywords}

Pericardium/agenesis; Pericardioectomy; Echocardiography/methods; Gestation; Parturition.
Physical examination revealed systolic murmur in basal foci $(2+/ 6+)$ and sporadic episodes of pulse irregularity. Electrocardiography showed bradycardia with sinus arrhythmia, right axis deviation (110 degrees), PRi of $200 \mathrm{~ms}$, poor R progression in precordial leads, and $\mathrm{T}$ inversion from V1 to V5 (Figure 1).

Posteroanterior chest radiography showed displacement of the cardiac axis to the left with no tracheal displacement, and right border of the heart at the spinal level (Figure 2).

Transthoracic echocardiography revealed unusual acoustic windows, posterior and superior deviation of the heart, atria and ventricles with "tear drop" appearance in the paraesternal view (Figure 3A) and lateralization of the four-chamber apical view, with preserved biventricular systolic function (left ventricular ejection fraction through the Teicholz method: $68 \%$ ) (Figure 3B). Exaggerated cardiac mobility in the chest (pendulum-like movement of the heart) was observed, in addition to paradoxal interventricular septal motion. Although cardiac chambers appeared to be enlarged in the subjective analysis, linear dimensions were found to be preserved (left ventricle end-diastolic diameter: $43 \mathrm{~mm}$, left atrial anteroposterior diameter: $29 \mathrm{~mm}$, right ventricle basal diameter in the four-chamber apical view: $30 \mathrm{~mm}$ ). No other structural changes were found (Video 1).

The patient's diagnosis was established 10 years ago, during outpatient cardiological assessment, due to complaints of frequent palpitations and dyspnea on moderate exertion. Results for chest radiography, electrocardiography, echocardiography, and magnetic resonance imaging of the chest were compatible with the diagnosis of complete pericardial agenesis.

One year after diagnosis, the patient was admitted due to typical chest pain and syncope. Cardiac catheterization was performed, with no

Mailing Address: Carolina de Souza Galvão

Hospital Universitário da Universidade Federal do Maranhão - Ecocardiografia

Rua Barão de Itapari, 227. Postal Code: 65020-070, São Luís, MA - Brazil.

E-mail: carolinasouza195@gmail.com 


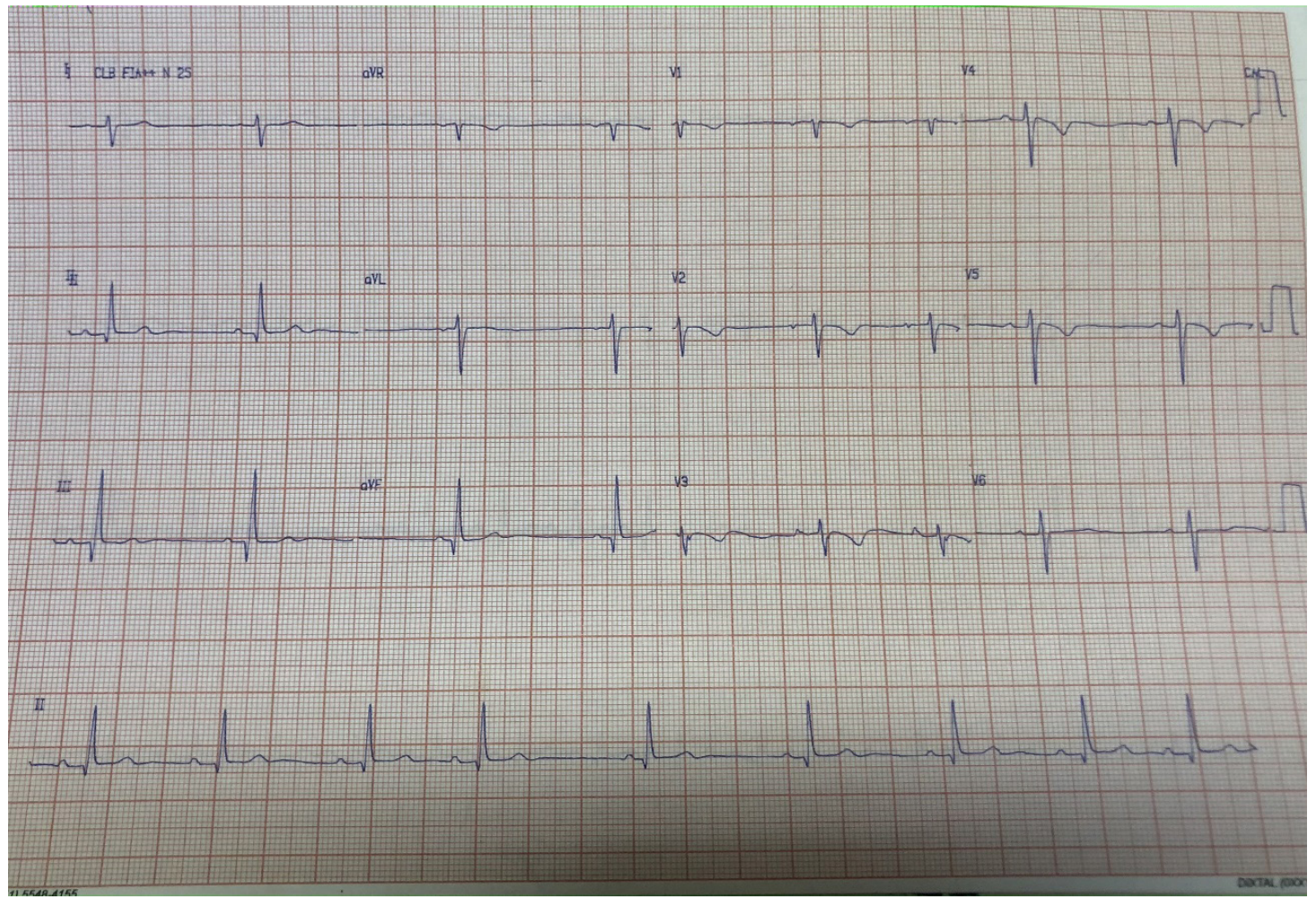

Figure 1 - Electrocardiogram: bradycardia with sinus arrhythmia, axis deviation to the right (110 degrees), PR interval: 200ms, poor progression of $\mathrm{R}$ in precordials and inversion of $\mathrm{T}$ from $\mathrm{V} 1$ to $\mathrm{V} 5$.

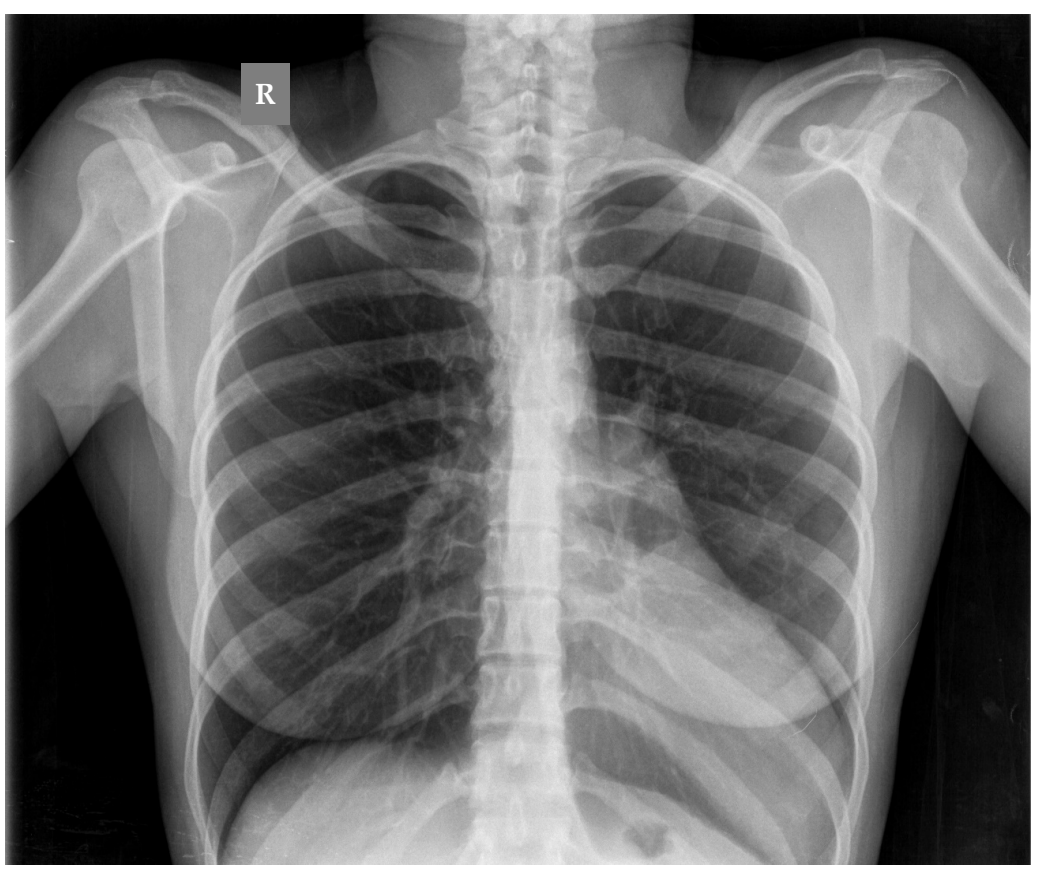

Figure 2 - Posterior anterior chest radiography: displacement of the cardiac axis to the left without tracheal deviation and the right border at the level of the spine. 


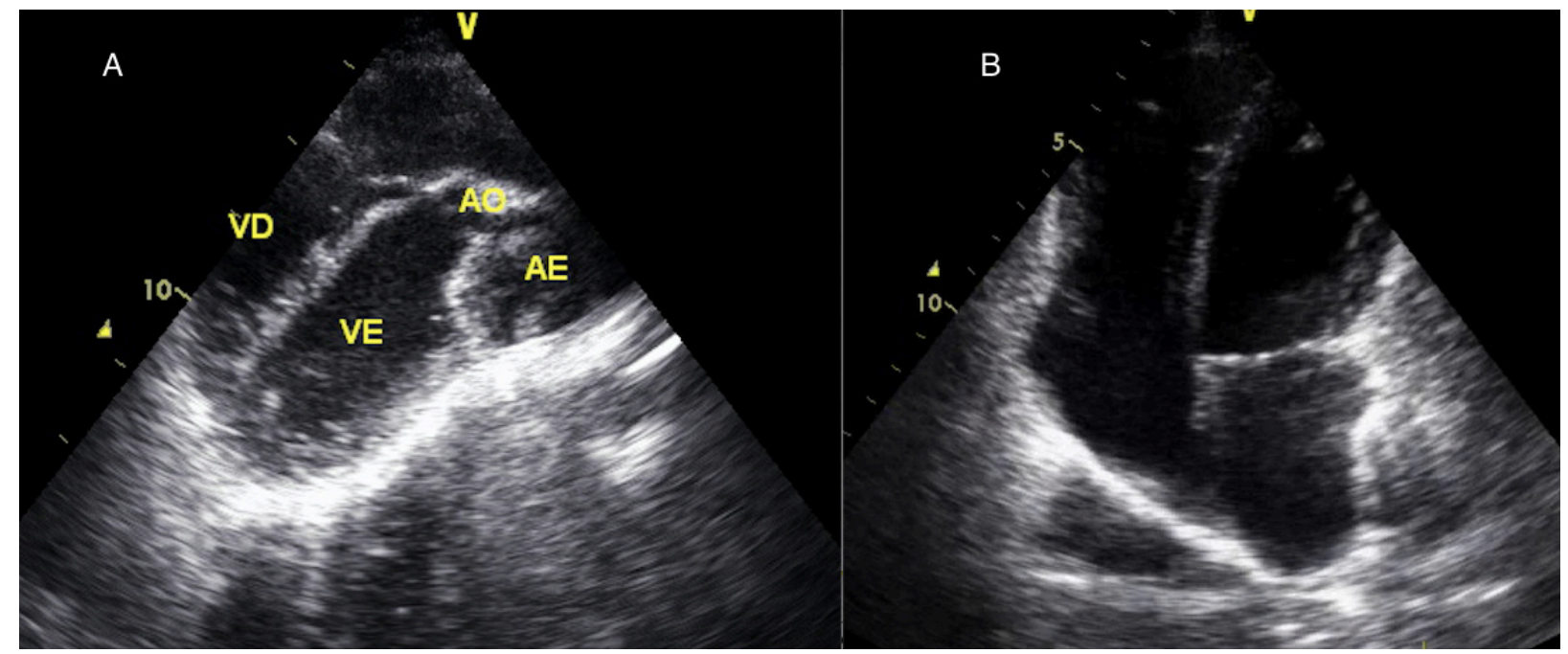

Figure 3 - Transthoracic echocardiogram: (A) enlarged ventricles and elongated atria appearing as a "teardrop" shape in the parasternal cut. (B) lateralization of the 4 -chamber apical cut.

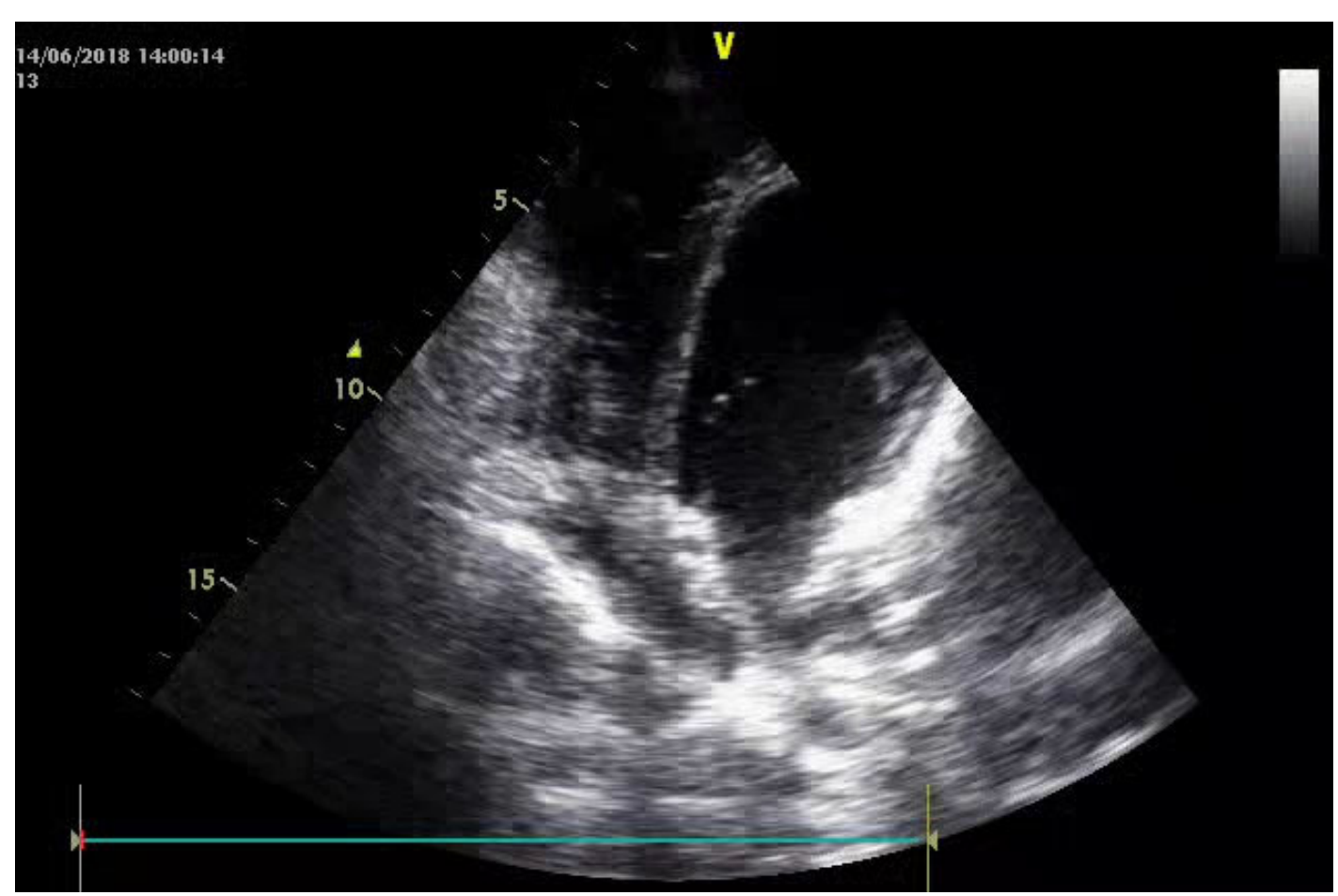

Video 1 - URL: http://ijcscardiol.org/supplementary-material/2021/3404/2021-0007-video-1.mp4 
evidence of coronary obstructions or intracardiac shunts, 24-h holter did not show significant changes, and electrophysiological study revealed preserved stimulus conduction and no induction of ventricular tachycardia. In that occasion, due to the technical difficulty associated with surgical reconstruction of the pericardium in complete pericardial agenesis and to non recurrence of these symptoms, the patient was discharged with the proposal of continuing outpatient follow-up with the attending cardiologist. However, the patient had irregular follow-up and only returned to the service when pregnancy was already advanced.

During hospitalization in the obstetrics ward, the patient did not have records of previous examinations for analysis. Neither computed tomography nor magnetic resonance imaging was requested during this hospitalization, given the uncertain safety of these tests during pregnancy.

Since it is an extremely rare disease, there is no consensus in the literature on the mode of delivery and on indication for sterilization in women with pericardial agenesis. The patient underwent a cesarean section after discussion with the multidisciplinary team, and she refused to undergo tubal ligation. The delivery was uneventful, and the immediate postoperative period was spent in the intensive care unit. The neonate was born healthy, with a gestational age of 37 weeks and 3 days, Apgar scores: 9/9 at 1 and 5 minutes, without congenital malformations, and the patient was instructed to continue outpatient follow-up with the cardiologist.

\section{Discussion}

The most accepted embryologic theory for the occurrence of pericardial agenesis is defective fusion of pleuropericardial membranes, with incomplete closure of the pleuropericardial cavity. ${ }^{5}$ This abnormality is supposed to result from early atrophy of left common cardinal vein, also known as the left duct of Cuvier, leading to hypoperfusion in these membranes. ${ }^{2}$

Pericardial agenesis may be classified into complete absence of the entire pericardium, absence of the right or left portion of the pericardium, or a partial, foramen-like defect. ${ }^{4,6}$ Complete bilateral absence of the pericardium is the rarest presentation, accounting for $9 \%$ of the cases, whereas complete absence of the left pericardium is the most reported one.,8 This classification has prognostic implications, with incomplete defects being more associated with herniation of cardiac structures. There are reports of three patients with partial absence of left pericardium with documented sudden death. ${ }^{6}$

Other congenital malformations should always be investigated, since there is a reported $30 \%$ of association with interatrial septal defects, patent ductus arteriosus, tetralogy of Fallot, bicuspid aortic valve, valve stenosis or prolapse. ${ }^{5}$

Complete absence of the pericardium is associated with hypermobility of cardiac structures and uncontrolled ventricular filling. This rare diagnosis is usually obtained in the postoperative context of thoracic surgical procedures or autopsy, given its benignancy and asymptomatic or oligosymptomatic presentation, with reports of atypical chest pain, dyspnea or trepopnea, which are manifestations of unknown origin, although they were probably related to significant dislocation of the heart and torsion of great vassels.5,9 In the case reported here, the diagnosis was established during outpatient cardiologic assessment due to symptoms of palpitations and dyspnea on moderate exertion.

The diagnosis of pericardial agenesis may be made through electrocardiography, chest radiography, echocardiography, computed tomography, and magnetic resonance imaging of the chest, the last of which is the most specific one. ${ }^{8}$ The most typical findings on electrocardiography are right axis deviation, blockade of the right branch, bradycardia, and poor R progression in precordial leads, and, more rarely, changes in segments ST and T waves. ${ }^{1,9}$ On chest radiography, the signal described as snoopy (displacement of the cardiac silhouette to the left, blurring of the right border of the heart, enlargement of its left border, and radioluscency between the left pulmonary artery and the aorta) was described in patients with complete pericardial agenesis. ${ }^{10-12}$

Echocardiography is an essential tool to suggest the diagnosis, making it possible to differentiate between complete and partial pericardial agenesis. However, it represents a technical challenge, due to hypermobility of cardiac structures and unusual echocardiographic windows. ${ }^{5}$ Paradoxal interventricular septal motion, apparent increase in right ventricle size, lateral deviation of apical windows, and "tear-drop"shaped chambers (enlarged ventricles and elongated atria) are possible findings that in complete pericardial agenesis, differently from partial agenesis, which is characterized by herniation of cardiac structures., ${ }^{2,13}$ 
Magnetic resonance imaging or computed tomography of the chest are more specific tests, and should be performed in the case of a strongly suggestive diagnosis, the most recommended of which magnetic resonance imaging, which is considered the gold standard, because it provides a better definition of structures, enables the detection of microinfarctions, is not associated with radiation, and does not require contrast media. ${ }^{5,11}$ The performance of these tests during pregnancy is not exempt of risks to the fetus, since magnetic resonance imaging, considered the preferred method over chest tomography, may be associated with hearing damage, tissue heating, and cell migration defects. ${ }^{14}$

The findings that may be found in the main imaging methods in patients with complete and partial pericardial agenesis are shown in table $1 .{ }^{5}$

Although the diagnosis of complete agenesis is associated with benignancy, ${ }^{8}$ the patient reported here had a history of admission due to typical chest pain and syncope, with more specific complementary tests to assess coronary heart disease and cardiac arrhythmia within reference range.

Table 1 - Imaging methods in complete and partial pericardial agenesis.

Chest radiography

Levoposition of the heart

Loss of the right cardiac border

Prominent left cardiac border

Interposition of the lung between the aorta and the main pulmonary artery

Interposition of the lung between the diaphragm and the heart

Prominent main pulmonary artery

$\begin{array}{cc}+ & +/- \\ + & +/- \\ +/- & + \\ + & +- \\ +/- & - \\ + & +/-\end{array}$

Echocardiography

Unusual acoustic windows

Cardiac hypermobility

Swinging heart motion

"Tear drop" appearance

Regional left ventricular bulging

Dilated left atrial appendage

Paradoxal interventricular septal motion

Right ventricular dilatation

Tricuspid regurgitation

\begin{tabular}{|c|c|}
\hline+ & $+/-$ \\
\hline+ & - \\
\hline+ & - \\
\hline+ & - \\
\hline - & + \\
\hline - & + \\
\hline+ & $+/-$ \\
\hline+ & $+/-$ \\
\hline+ & $+/-$ \\
\hline+ & - \\
\hline+ & + \\
\hline- & $+/-$ \\
\hline$+/-$ & $+/-$ \\
\hline - & $+/-$ \\
\hline - & $+/-$ \\
\hline$+/-$ & $+/-$ \\
\hline
\end{tabular}

Magnetic resonance imaging of the chest

Unusual displacement of the heart

Absence of pericardium between tissue and fat

Interposition of the lung between the heart and the diaphragm

Interposition of the lung between the aorta and the main pulmonary artery

Cardiac wall notch

Cardiac herniation

Left lung hypoplasia

$$
\begin{aligned}
& +- \\
& +/ \\
& + \\
& +-
\end{aligned}
$$$$
+
$$$$
+-
$$

source: adapted from Centrola $M$, Longo M, Cremoseni $G$, Marconi M, Danzi GB5. 
There are no established recommendations for surgical intervention in patients with pericardial agenesis, but this strategy is more used in the partial symptomatic presentation of the disease, due to the greater risk of herniation of cardiac structures. Pericardioplasty represents the most used surgical technique in these patients. ${ }^{6}$

The physiology related to normal pregnancy is mainly characterized by progressive increase in plasma volume with stabilization up to the end of the third trimester, increased cardiac output from 30\% to 50\% of baseline, increased heart rate (nearly 12 beats per minute), and reduced systemic vascular resistance with increased prostacyclin levels. During childbirth, more hemodynamic changes are expected, such as increases in blood pressure and cardiac output; furthermore, the more significant these increases are, the stronger and the more frequent uterine contractions are, and the greater patient's anxiety and pain are. The most critical phase occurs soon after birth, considering the significant increase in blood pressure and blood volume, related to the effects of placental detachment (increased vascular resistance, decompression of the inferior vena cava, and release of the volume that was present in the uterus). ${ }^{15}$

Considering that the main function of the pericardium is restricting cardiac volume, as well stabilizing the positioning of cardiac structures in the chest and producing chemicals that affect the tone of coronary arteries, ${ }^{16}$ pregnant women with pericardial agenesis are supposed to have higher risk of complications than other pregnant women, especially during childbirth and the immediate postpartum period, when there is a significant increase in cardiac volume and cardiovascular demand. Given the rarity of pericardial agenesis, there are no specific recommendations in the literature with regard to its prognosis in pregnancy.

The choice of the mode of delivery in patients with heart disease is still a controversial topic in the absence of severe heart failure, Eisenmenger's syndrome, severe aortic stenosis, and ascending aorta dilation (> $45 \mathrm{~mm})$, which are the formal recommendations of cesarean delivery, as well as specific obstetric indications. ${ }^{17}$ A study conducted in 28 countries, based on the Registry on Pregnancy and Cardiac Disease from 2008 to 2011, included 1321 pregnant women with heart disease and compared vaginal delivery and planned cesarean delivery. No significant differences were observed in maternal mortality, postpartum heart failure and hemorrhage, and Apgar scores, but birth weight and gestational age were lower in women who delivered by cesarean section. ${ }^{18}$ In the present case, an elective cesarean section was chosen after a meeting with a multidisciplinary team, when the risk and benefits of vaginal delivery were pondered, as well the possibility of evolving to emergency cesarean section in a patient without pericardium for the appropriate control of cardiac volume.

Female sterilization is part of a contraceptive strategy in patients at high cardiovascular risk and may be lead to great psychological impact, late contraceptive failure (especially in young women and when the procedure is performed concomitantly with cesarean section), and increased likelihood of an ectopic pregnancy. A levonorgestrel-releasing intrauterine device (IUD) and the Implanon subdermal implant are other options in patients with heart disease, and are even considered more effective than sterilization, although requiring additional care and additional costs. ${ }^{19}$ Considering the risks of a new pregnancy in the case reported here, tubal ligation was the first contraceptive method of choice; however, the patient refused sterilization, and the chosen contraceptive method was a levonorgestrel-releasing IUD.

\section{Author contributions}

Conception and design of the research: Galvão CS, Abreu SLL, Carvalho MLS. Acquisition of data: Galvão CS, Abreu SLL, Serra JCU. Writing of the manuscript: Galvão CS, Carvalho MLS, Silva GN. Critical revision of the manuscript for intellectual content: Abreu SLL, Carvalho MLS, Serra JCU, Silva GN, Leite ABSS.

\section{Potential Conflict of Interest}

No potential conflict of interest relevant to this article was reported.

\section{Sources of Funding}

There were no external funding sources for this study.

\section{Study Association}

This study is not associated with any thesis or dissertation work.

\section{Ethics approval and consent to participate}

This article does not contain any studies with human participants or animals performed by any of the authors. 


\section{References}

1. Abbas AE, Appleton CP, Liu PT, Sweeney JP. Congenital absence of the pericardium: case presentation and review of literature. Int J Cardiol. 2005;98(1):21-5.

2. Connolly HM, Click RL, Schattenberg TT, Seward JB, Tajik AJ. Congenital absence of the pericardium: echocardiography as a diagnostic tool. J Am Soc Echocardiogr. 1995;8(1):87-92.

3. Yamano T, Sawada T, Sakamoto K, Nakamura T, Azuma A, Nakagawa M. Magnetic resonance imaging differentiated partial from complete absence of the left pericardium in a case of leftward displacement of the heart. Circ J. 2004;6(5)8:385-8.

4. Van Son JA, Danielson GK, Schaff HV, Mullany CJ, Julsrud PR, Breen JF. Congenital partial and complete absence of the pericardium. Mayo Clin Proc .1993; 68(8): $743-7$

5. Centrola M, Longo M, De Marco F, Cremonesi G, Marconi M, Danzi GB Does echocardiography play a role in the clinical diagnosis of congenital absence of pericardium ? A case presentation and systemic review. J Cardiovasc Med. 2009;10(9): 687-92.

6. Lopez David, Asher Craig R. Congenital Absence of the Pericardium. Prog Cardiovasc Dis. 2016;59 (4):398 - 406.

7. Bouchard M,. Hoschtitzky A, Gatzoulis M. Diagnosis and management of congenital absence of pericardium: a case report. Eur Heart J. 2019; 3(4): 1-5.

8. Nasser WK. Congenital diseases of the pericardium. Cardiovasc Clin. $1976 ; 7(3): 271-86$

9. Gatzoulis MA, Munk MD, Merchant N, Van Arsdell GS, McCrindle BW, Webb GD. Isolated congenital absence of the pericardium: clinical presentation, diagnosis, and management. Ann Thorac Surg. 2000;69(4):1209-15.
10. Han J, Xiang H, Ridley WE, Ridley LJ. Snoopy sign: Congenital absence of the left pericardium. J Med Imaging Radiat Oncol. 2018; Suppl 1:47.

11. Palomino AB, Estrada AP, Crespin MC, Fuertes DG. Congenital complete absence of pericardium in a young woman with non-specific symptoms. Rev Port Cardiol. 2014;33(4):249.e1-e5.

12. Morgan JR, Rogers AK, Forker AD. Congenital absence of the left pericardium: clinical findings. Ann Intern Med. 1971;74(3):370-6.

13. Candan I, Erol C, Sonel A. Cross sectional echocardiographic appearance in presumed congenital absence of the left pericardium. Br Heart J 1986;55(4):405-7.

14. Rocha APC, Carmo RL, Melo RFQ, Vilela DN, Leles-Filho OS, Costa-Silva L. Avaliação por imagem de condições não obstétricas na gestação: o que todo radiologista deve saber. Radiol Bras. 2020;53(3):185-94.

15. Picon JD, Sá AMPOA. Alterações hemodinâmicas da gravidez. Revista da Sociedade de Cardiologia do Rio Grande do Sul. 2005.

16. Zipes DP, Libby P, Bonow RO, Mann DL, Tomaselli GF. Braunwald's Heart Disease: A textbook of cardiovascular medicine. 11nd ed Philadelphia:Elsevier; 2019,

17. Zagrosek VR, Hesselink JWR, Bauersachs J, Lundqvist CB, Cífkova R, Bonis M, et al. 2018 ESC Guidelines for the management of cardiovascular diseases during pregnancy. Eur Heart J. 2018;39(34):3165-241.

18. Ruys TP, Roos-Hesselink JW, Pijuan-Domènech A, Vasario E, Gaisin IR, Iung B, et al. Is a planned caesarean section in women with cardiac disease beneficial? Heart. 2015;101(7):530-6.

19. Thorne S, MacGregor A, Piercy CN. Risks of contraception and pregnancy in heart disease. Heart 2006;92(10):1520-5. 